\title{
Gritos de la tierra. Historias de vida de los mineros del carbón*
}

\section{Daniel González Cortés}

...es sumamente fácil hablar de la vida del minero, de su familia y de sus peripecias en las luchas cotidianas. Es fácil, repito, hacerlo desde los escritorios, dramatizarla y darle sabor y colorido según las circunstancias que la rodean. Yo escribo como viejo minero de la región carbonífera de Coahuila; en donde el trabajo dentro y fuera de la mina es agobiante y extenuante, máxime cuando la fuente minera está desposeída de la técnica y seguridad necesarias para la vida del minero. ${ }^{1}$

\section{Introducción}

Las diferentes investigaciones y estudios referentes a la clase obrera en México, si bien innumerables y de extensa práctica dentro de la tradición en las ciencias sociales, están obteniendo un impulso renovador con el aporte de las fuentes orales, las cuales pretenden acercarse a la esencia de los hechos, tanto individuales como sociales; en otras palabras, a la historia de los mismos protagonistas.

Estas fuentes ayudan a comprender más la parte cotidiana de la vida del hombre en el trabajo, familia, sindicato, barrio, mercado, festejo, escuela, diversiones..., en otras palabras, el tiempo laboral y el tiempo libre con el que los hombres y mujeres viven; darnos cuenta de que se trata de una interpretación diversa del mundo y de la vida.

Ajena a la historia formal, en donde el personaje y los grandes hechos determinan los grupos, se escapa de la cultura misma una historia sumergida, casi clandestina, en donde los actores principales ponen en escena su concepción del mundo, la vida, la sociedad y la naturaleza.

\footnotetext{
* Ponencia presentada en el Primer Encuentro de Historiadores Orales de América Latina y España. Instituto de Investigaciones Dr. José María Luis Mora, México, septiembre de 1988.

Todas las citas son testimonios orales y escritos recopilados a lo largo de meses en la cuenca carbonífera de Coahuila. Por el tiempo otorgado, por haber compartido sus recuerdos y parte de su vida, doy agradecimiento a las siguientes personas: Manuel J. Santos, Antonio Garcia Moreno, Abdenago Fausto García, Gabriel Almanza, Bernardino Ayala, Manuel Chacón, Gilberto Cota, Agapito Maltos, Luis Muñoz, Ernesto Pérez, Jesús Rea, Tomasita Rodríguez, Ramón Banda, Atilano Campos, Inocencio Guerrero, Eligio Terán, Fructuoso González y al doctor Enrique Guajardo Reyes; al personal de Industrial Minera México, S.A., de Minerales Monclova, S.A., y de la Cia. Minera Guadalupe.
} 
El presente escrito no trata de convencer de las bondades que tiene o puede ofrecer la información oral para reconstruir hechos o historias de vida. De lo que se trata es de discernir cómo las historias de vida pueden aportar información para entender más la cosmovisión de otros grupos y clases sociales.

En las ciencias sociales, y en particular en la antropología, las historias de vida siguen siendo de carácter descriptivo y se encargan de los datos biográficos como punto de partida para entender la forma de actuar y ser del individuo y de la cultura a la que pertenece.

En el caso de la cultura de los mineros del carbón, las historias de vida realizadas determinaron dos ejes en los cuales profundizó más la gente: el enclave o "mineral" y el significado del trabajo en las minas del carbón.

Me voy p'al pueblo, hoy es mi dia...

En un primer momento la historia de vida es una descripción y una evocación del recuerdo que un individuo hace de sus propias acciones, vivencias y creencias. No hay historias de vida que no revivan a la ascendencia y al cómo se llegó a la matria o tierra prieta.

Aquí les voy a prestar un diario que fui escribiendo en el transcurso de mi vida [...] aquí relato yo, si llovió o no llovió, el tiempo que hizo, $\mathrm{mi}$ primer día de trabajo, los accidentes que sufrí, cuándo me casé y también lo que me contaban mi padre y mi abuelo [...] Ellos vinieron enganchados a trabajar en estas minas a principios de siglo; la población, me cuentan, se formó con gente de muchos estados del país, incluso de China y Japón [...] por eso es que en esta región no hay una cultura en especial o que tenga la región sus tradiciones, cada quien trajo lo suyo su aportación y se formó esto y fue creciendo en una forma especial $[\ldots]$

La mayoría de las poblaciones mineras del norte de México datan de principios de siglo, cuando el gobierno mexicano dio facilidades de inversión al capital extranjero; en el caso de Coahuila, la familia Guggenheim, dueña y fundadora de la Compañía Minera ASARCO (American Smelting and Refining Company), recibió el 9 de octubre de 1890 una concesión de Porfirio Diaz para abrir tres plantas fundidoras en México. Escogieron la ciudad de Monterrey como lugar estratégico para establecer las fundiciones, ya que las conexiones ferroviarias les permitían transportar a Monterrey el carbón de las poblaciones de la cuenca.

En Rosita se explotaron cinco tiros: el número 1 que se localizó ahí mismo en la Rosita; [...] simple y sencillamente se descubrió la mina, y en la necesidad de hacer un pueblo le pusieron una plaza, y alrededor un palacio municipal, un teatro, al otro lado la iglesia, el

2 La cuenca carbonifera comprende las poblaciones de Nueva Rosita, San Juan Sabinas, Palau, Melchor Múzquiz, Rancherías, Esperanzas, Barroterán, Agujita y Cloete, productoras del $\mathbf{9 7 . 9 \%}$ de carbón mineral. Todas estas poblaciones al norte del estado de Coahuila. 
sindicato, un restaurante y allí empezó, echándole calles por donde quisieron y hacer las casas en donde les dio la gana; [...] se acabó el mineral y cerraron los tiros $2,3,4$ y 5 ; la ASARCO, al ver que se estaban agotando los mantos, dio principio a la perforación del tiro número 6; [...] se hizo alrededor del tiro 6 una sección de corralones hechos para enganchados mexicanos, otros para chinos; hacia arriba en la lomita se conformó la colonia Americana, que era para puros norteamericanos, después vinieron ingenieros que no pertenecian al rango norteamericano, jefecitos o gente como esa y se formó la colonia Independencia, se hizo para gente de más arriba que los mineros; habia un grupo de trabajadores que decidieron hacer sus casas hasta la orilla del rio, buscando agua y buscando también terrenos de cultivo y así se creó la colonia Rubirosa; es una colonia de trabajadores, de gente pobre, otra colonia cercana es la colonia Zaragoza, pero también para trabajadores de bajo rango.

La geografía de una población minera está caracterizada por el papel de los trabajadores adscritos a la organización social del trabajo. La jerarquia que predomina en el trabajo predomina también fuera de él: diversiones, escuelas, colonias, centros de compras, forma de vestir, comida, transporte, entre otros, están caracterizados por este rango de relaciones descendentes.

Este modelo empresarial, conocido en términos sociológicos como "enclave" se sustentó en un sistema de organización de la producción minera caracterizado por la implantación de grandes empresas monopólicas de capital y tecnologia extranjeras. El enclave se apoyó en una serie de barreras: la discriminación étnica, tanto en el trabajo como en el espacio urbano, la formación de un mercado de trabajo caracterizado por la inestabilidad y la heterogeneidad y el recurso del paternalismo frente a toda demanda obrera.

La discriminación étnica no sólo caracterizó la división del trabajo en las minas y plantas metalúrgicas, sino que también se plasmó en una política de salarios diferenciados según nacionalidades, y en una concepción de segregación espacial en el trabajo urbano de los "minerales" y en el acceso a determinados servicios públicos. Todavia hoy en día, en estas poblaciónes es fácilmente reconocible la línea divisoria de las colonias, entre empleados y directivos empresariales y los barrios de las familias mineras mexicanas. ${ }^{4}$

En las poblaciones mineras mexicanas del norte de México los pobladores, al referirse al lugar en donde viven, hablan de "mineral"; este concepto refleja una forma de vida determinada en todos los niveles por la empresa minera; de hecho, todo lo que se hace o deja de hacer está relacionado con ésta. El monopolio que tiene la empresa sobre el trabajo y el pueblo -e incluso sobre la tierrarepercute en la población de dos maneras: la necesidad de com-

Daniel González Cortés, "Ta'oscuro el panorama" (relatos sobre los mineros del carbón) Monografias obreras, Centro de Investigaciones y Estudios Superiores en Antropologia Social (CIESAS), México, 1987. (Cuadernos de la Casa Chata, 142).

4 Juan Luis Sariego Rodríguez, "La cultura obrera en crisis. Aproximación a algunos elementos de la identidad de un grupo obrero", Coloquio sobre cultura. obrera, CIESAS, México, 1987 (Cuadernos de la Casa Chata, 149). 
prar un terreno para habitarlo y la carencia de alternativas ocupacionales;

ahi tiene usted que a los trabajadores que según la empresa son revoltosos los saca del trabajo y con éste pierden hasta el derecho a la casa, y para buscar aquí está dificil pues todo lo tiene la compania minera; [...] esto sólo pasa en los minerales, [...] se acaba lo que saca uno de la tierra, la empresa decide irse y todo lo que estaba ahi se va, hasta la gente.

El abastecimiento de servicios públicos (agua potable, alcantarillado y drenaje, vías públicas y espacios libres, limpieza de calles, alumbrado, pavimentación, administración y educación) por algún tiempo estuvo a cargo de la empresa casi regalados, pero no siempre habia. En la actualidad, según las compañias mineras, el gobierno debe proporcionarlos, pero éste es de la opinión contraria; lo que hace que se esfumen las responsabilidades, pues ninguna de las partes las asume.

El pueblo tiene y conserva aún una situación especial, la de mineral. Esto parece que influye en la forma en que se distribuyen los gastos públicos; probablemente los impuestos que pagamos, porque aqui todos pagamos impuestos, no revierten sobre el pueblo sino sobre el estado, [...] si no, no se explicaría por qué otros pueblos más pequeños tienen mayores servicios de urbanización y nosotros no.

Esta es la visión de los mineros que han vivido toda su vida en un mineral y no quieren, por ningún motivo, que sus hijos ni sus descendientes sean lo que ellos han sido, pues su trabajo es peligroso y mal pagado. Por otra parte, vivir en una población minera es tragarse la angustia diaria de no tener un lugar seguro en donde vivir y el miedo a que se termine la fuente de trabajo y con esto, el mineral pase a ser un pueblo fantasma.

\section{Minero soy; \\ mi sino, sufrir; \\ en las entrañas \\ de las montañas \\ he de morir}

Cuando no se conoce lo que es el trabajo minero y nos hablan de él, por lo general se nos viene a la mente la idea del hombretopo o gambusino que anda probando suerte y en busca de aventuras que le darán grandes ganancias. La realidad es diferente, la minería del carbón es uno de los trabajos que se desenvuelven en condiciones de lo más difíciles y peligrosas.

Dadas las condiciones de inseguridad e insalubridad, características de las galerias y de los frentes de extracción y explotación, la falta de ventilación, la oscuridad, filtraciones e inundaciones, y a los riesgos de accidentes, derrumbes y explosiones, abundan las enfermedades profesionales, como la silicosis, la sordera y el reumatismo. Cuando los trabajadores mineros narran algo sobre su trabajo, no falta alguna desgracia personal o colectiva; se tienen 
registrados en la mente, los días, número de muertos, tipo de accidentes, número de explosiones y derrumbes:

a los 14 años comencé a trabajar en la mina, antes no había nada de ir al sindicato a pedir permiso ni formar cola en la empresa para que lo contrataran a uno junto con otras personas; un día llegamos hasta la puerta de la planta, y nos dijeron que nos presentáramos en la mina al día siguiente; [...] me integré al trabajo de carbonero sin nada de conocimiento más que lo que hablaban mi padre y mis hermanos, era algo así como comodín que tenía que hacerle de todo mientras me daban mi puesto como ayudante de alguna cosa; me entrevistó un ingeniero que estaba encargado de la mina, diciéndome: ¿dónde ha trabajado usted?, y le respondí que no sabía nada de la minería, lo que sé es de la labor [del campo], sé manejar el azadón, el hacha, el corte, la pizca del algodón, de todo sé hacer señor. Ah..., entonces yo creo que sí puede. Al pasar algunas semanas de prueba y de mucho trabajo me encontré con un familiar, él sabia lo duro del trabajo del campo y me dijo: Oye... ¿qué tal el trabajo en la mina? Yo le dije: No... pos está duro, aparte de la chinga del trabajo, no me logro acostumbrar a la oscuridá, siento como que me falta el aire, se suda mucho, la vigilancia está tras de uno, hasta parece uno preso, no dan tiempo para comer, ni para ir a miar. ¿Te das cuenta que chingón trabajo?, pero la necesidad es cabrona y me tuve que quedar a trabajar; y ya me acostumbré a todo eso [...] A lo que nunca me he podido acostumbrar es al miedo que da la mina; no es miedo de espanto, sino es tener miedo al peligro, siempre lo tiene uno constante, pues los peligros abundan; nunca se sabe qué le puede pasar a uno, no obstante que los ingenieros dicen que a nada tenemos que temer pues hay mucha seguridad y que ya no es como antes, que el trabajo ya está mecanizado; pero nosotros lo sentimos y vemos a diario, si no le toca a uno el peligro le toca al compañero de junto.

Los riesgos de trabajo en las minas de carbón no han dejado de ser problema para las compañias e instituciones, que están dedicadas a prevenir accidentes y sugerir mejoras en la seguridad industrial. El Instituto Mexicano del Seguro Social (IMSs), la Secretaría del Trabajo y Previsión Social, la Organización Internacional del Trabajo y las Comisiones Mixtas de Seguridad e Higiene, reconocen que de todos los trabajos existentes en México la minería, sobre todo las minas de carbón, en relación con las minas metálicas, es la que presenta más alto riesgo por sus condiciones de trabajo.

La medicina laboral que se realiza en la región ha prestado poca atención a las enfermedades de trabajo, es decir, aquellas enfermedades que sin mediar accidentes se generan entre los mineros después de largos periodos de vida laboral y directamente relacionadas con las condiciones del trabajo minero.

De hecho está claro que el número de accidentes en las minas ha aumentado desde que el IMSS opera en la cuenca, en especial el número de lesiones leves que pueden provocar una incapacidad temporal. Esta es una de las cosas que comúnmente recriminan al IMSS las empresas, y muy probablemente se debe a dos causas: por

${ }^{6}$ En el Departamento de Medicina Preventiva de la Clínica Regional del IMSS en Nueva Rosita, Coahuila, el $70 \%$ de los casos tratados se relacionan con accidentes de trabajo. 
una parte, al ser el IMSS quien decide la incapacidad de un trabajador y no la empresa, es probable que determinadas incapacidades antes no consideradas por las empresas pasen ahora a serlo. Por otra parte, sin duda el minero percibe al IMSS como una instancia que puede entender y defender mejor sus intereses y ahi se escuda para hacer valer su incapacidad para el trabajo después de una de esas lesiones leves.

El IMSs llega a la región carbonífera en 1970, debido a la presión de la población por las explosiones de las minas 2 y 3 de Barroterán, en las que murieron dos turnos de mineros.

La mina 2 era una mina angosta por ser bastante antigua; y no se preparó la mina adecuadamente para el sistema moderno y de frentes largas que entró en 1967 con la mecanización. Se empezaron a expandir como pulpo las labores, y mientras más se abrían, disminuía la ventilación; con tanto túnel, el ventilador no daba el aire suficiente; además, los túneles de regreso los hicieron de un metro, para ahorrar; y la explosión la ocasionó la chispa de un motor que flameó.

Las explosiones que ha habido -30 en el presente siglo con más de 2000 muertos - se debieron a falta de seguridad.

Antes de la llegada del IMSs la gente que trabajaba para las compañías tenía derecho al hospital de ésta; a sus familiares los atendían en la clínica del sindicato y el resto de la población tenía que rascarse con sus propias uñas.

Las heridas en manos, piernas y otras partes del cuerpo se intensifican más en los turnos nocturnos, pues la fatiga lleva a que haya más lesiones. Las enfermedades psíquicas constituyen otro factor que caracteriza el trabajo del minero. Las fobias son causadas por tensiones en el trabajo y por estar en constante peligro:

entre los trabajadores mineros no he visto casos de esquizofrenia, en cambio de neurosis sí, por ejemplo las fobias. En una ocasión vino la esposa de un trabajador sumamente angustiada y me dijo: Oiga doctor, no sé qué le pasa a mi esposo; tiene miedo, sobre todo a los lugares oscuros y a estar encerrado; y trabaja en la mina. Llega él y le pregunto: ¿Tienes miedo a la mina?, y me dice que no, que está acostumbrado, que nunca le ha tenido miedo a la mina. Nos podemos imaginar cuál es el origen del problema y su desencadenamiento; lógicamente son individuos que están constantemente en peligro; en este caso, hacía dos o tres días que hubo un "caido" que le llaman; es decir, se vinieron abajo muchas piedras que mataron a dos individuos; y el paciente, a los dos días comenzó a tener terror a los lugares encerrados. Se le desencadeno, y cuando llegaba a la casa y veia un clavo decía: quítalo, quitalo porque se van a cortar los niños y les va a dar tétanos y se van a morir; pasaba por una telaraña y decía: Ahí debe haber una araña, mátala porque va a picar a los niños y se van a morir. La mujer le decía: Ve a comprar esto. Y decía: No, porque está muy oscuro y me pueden asaltar y matar. Decía que no le alcanzaba el aire, respirando hondo, estaba asustado; estos momentos de angustia le hacían llorar y decía: no sé qué me pasa. Todos estos son datos que demuestran la

7 De las explosiones con más cantidad de victimas tenemos: en 1899, la mina Hondo Núm. 6 con 300 muertos; 1908 , la mina Rosita Núm. 3 con 200 muertos; 1969, Barroterán con 153 muertos. Cf. González, op. cit. p. 138, cuadro de explosiones. 
fobia de la que son conscientes pero no podian gritar. El caso se repitió en esa ocasión en unos diez trabajadores, todos jóvenes.

Otro que padecía esto mismo hacía dos días habia visto cómo una máquina mataba a uno de sus compañeros. Estos padecimientos psiquicos son causados por las condiciones en que trabajan los mineros. Además, entre los mineros, se da un caso especial, si uno de los mineros se queda atorado en algún caido, o hay peligro por los gases, hasta el más miedoso se lanza para ayudarlo; hay mucha unión, algo muy especial: se lanza a sacarlo, aunque sepa que su vida está en peligro.

No obstante que la tecnología ha avanzado, las enfermedades pulmonares causadas por lo que inhalan, y las reumáticas, por el cambio brusco de temperatura, no han podido ser combatidas. Padecimientos de la piel como las tiñas son características del minero, quien transmite la enfermedad a toda la familia; el foco de infección es el lugar de trabajo. Las llagas en piernas y piés causadas por el sudor, por la rozadura de las botas de hule, son parte de los nuevos padecimientos que no reconocen las compañias.

\section{Nadic es profeta en su tierra}

El trabajador en estas zonas mineras es muy especial, diferente a los trabajadores de otras regiones de la república; el minero del carbón no tiene ambiciones y hasta llega a la mediocridad, es por eso que pasa el tiempo y el minero sigue igual, porque, por más dinero que se le pague si no se tiene cultura, educación y ganas de superarse, las cosas no cambian $[\ldots]$

Esta es la mentalidad de la mayoria de la gente hacia el minero y su descendencia, quienes son víctimas de una concepción errónea de lo que es la cultura. En este escrito, el término cultura reconoce que son las condiciones de la vida material (la existencia) las que determinan la conciencia de los hombres; esto es, que los planteamientos, las reflexiones, las concepciones que los hombres tengan en un momento dado, derivan de una situación objetiva en una sociedad concreta.

De todo el proceso cultural, modelado y moldeado, por el que los individuos pasamos cuando vivimos en sociedad, resulta una manera de ser, estar, pensar, sentir, creer, una situación concreta, histórica y social. En una sociedad las relaciones y las prácticas sociales se desarrollan en una serie de niveles y estructuras educativas, politicas, económicas y por medio de sus instituciones principales: familia, iglesia, escuela y organizaciones políticas, se trasmiten los valores, normas, aspiraciones y costumbres de esa sociedad.

En el caso de la cultura de los mineros del carbón, las respuestas históricas de este sector obrero, implican valores, comportamientos que apuntan - implícita o explícitamente hacia una visión del mundo-. Hablamos de respuestas porque, en sus diferentes expresiones, la cultura obrera tiene siempre el carácter de una alternativa o una resistencia frente al modelo de la cultura hegemónica burguesa. Subrayamos la dimensión histórica, porque las respuestas obreras se inscriben dentro de una experiencia capaz de unificar a los sujetos que la viven.

${ }^{8}$ Sariego, op. cit. 
Alcoholismo, ignorancia, hacinamiento, enfermedades, accidentes y demás males estructurales, se achacan al minero porque no son vistos como males sociales que salen del mineral y que son problemas de todo mexicano. La cultura del minero del carbón es más amplia que los estudios formales que se tienen sobre ella, pues está ligada a las costumbres y conocimientos que se transmiten de padres a hijos en una educación no formal; los conocimientos, actitudes, formas de vivir y de ser conforman la cultura a la que pertenecen. Para terminar con este escrito daré la despedida de un minero:

aunque sufri bastante en la mina, siento como si fuera ayer cuando por primera vez ingresé a ella; recuerdo a mis excompañeros, a todos los fallecidos, ya sea por enfermedad o por accidente, los llevo en mi memoria con un amor de hermanos y compañeros del mismo dolor, de la misma tierra que me vio nacer; recuerdo la mina que fue mudo testigo de mis fatigas y peligros que allí pasé.

\section{Glosario}

Caido: Derrumbe dentro de la mina, por lo general siempre son piedras o bloques de carbón del cielo.

Carbonero: Encargado de tumbar carbón y palearlo.

Cielo: Techo de la mina.

Enclave: Término que engloba aislamiento geográfico, carencia de todo con condiciones inapropiadas de vida y de trabajo.

Enganche: Los enganches se hacian a principios de siglo para llevar gente a trabajar a una zona minera.

Frente: Lugar de trabajo, lugar de extracción.

Galeria: Cañón de acceso a otro lugar.

Labor: Otra forma de decir "frente".

Mineral: Para designar un pueblo minero, en las poblaciones del norte de México. se utiliza esta forma. También tiene una acepción despectiva, ya que la población vive del trabajo de la única empresa a la cual está ligada en todas las fases.

Tiro: Túnel de entrada a la mina. Hay tiros en forma vertical y horizontal. 